\title{
RAINDROP IMPACT ON SAND: A DYNAMIC EXPLANATION OF CRATER MORPHOLOGIES
}

\author{
SONG-CHUAN ZHAO\#, RIANNE DE JONG, AND DEVARAJ VAN DER MEER
}

\begin{abstract}
As a droplet impacts on a granular substrate, both the intruder and the target deform, during which the liquid may penetrate into the substrate. These three aspects together distinguish it from other impact phenomena in the literature. We perform high-speed, double-laser profilometry measurements and disentangle the dynamics into three aspects: the deformation of the substrate during the impact, the maximum spreading diameter of the droplet, and the penetration of the liquid into the substrate. By systematically varying the impact speed and the packing fraction of the substrate, (i) the substrate deformation indicates a critical packing fraction $\phi^{*} \approx 0.585$; (ii) the maximum droplet spreading diameter is found to scale with a Weber number corrected by the substrate deformation; and (iii) a model about the liquid penetration is established and is used to explain the observed crater morphology transition.
\end{abstract}

Liquid droplet impact on a granular layer is very common in nature, industry, and agriculture, ranging from raindrops falling onto desert or soil to granulation in the production process of many pharmaceuticals. In spite of its commonness, the physical mechanisms involved in the impact of a droplet on sand did not attract much attention until recently [1-12, and the underlying physics is still largely unexplored. In contrast, droplet impact on a solid surface or a liquid pool has been studied extensively [13-15]. However, a granular substrate can act both solidlike and fluid-like [16] and many experiments have been conducted to reveal the response of a granular packing to a solid object impact [17- 23], where the intruder does not deform. Droplet impact on a granular substrate adds new challenges to the above: First, both the intruder and the target, not merely one of the two, deform during impact; second, the liquid composing the droplet may penetrate into the substrate during the impact and may, in the end, completely merge with the grains. These complex interactions between the droplet intruder and the granular target create various crater morphologies as reported in the literature [1, 2, 4, $, 1,12]$ [see Fig. 1 for examples]. An appealing and natural question is by what mechanism craters are formed and how this leads to the observed rich morphological variation. This is the main focus of the present work. Quantitative dynamic details, e.g., on the deformation of droplet and substrate in response to the impact, are necessary to gain insight about this issue. Previous dynamic measurements only regard the

\# Physics of Fluids Group, Faculty of Science and Technology, University of Twente, PO Box 217, 7500 AE Enschede, The Netherlands; E-mail: szhao@utwente.nl 


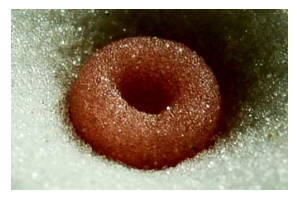

doughnut

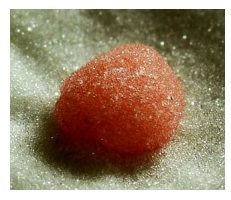

truffle

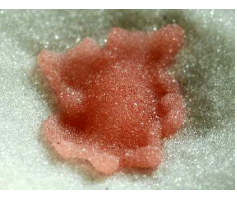

pancake

FiguRE 1. Various crater morphologies observed in the experiments are categorized as: doughnut, truffle, and pancake.

droplet spreading [2,4,5] or individual splashing grains [8], and a systematical study of the effect of the packing fraction of the substrate is still missing. In this paper, we perform dynamic measurements with high-speed laser profilometry and study the dependence of the dynamics on impact speed and packing fraction of the granular substrate. Following the discussion about the results of the substrate deformation and the droplet deformation and the underlying physics, a quantitative model is established which explains the observed crater morphologies in the end.

\section{EXPERIMENTAL METHODS}

A water droplet with diameter $D_{0}=2.8 \mathrm{~mm}$ acts as the intruder. The water droplet is pinched off from a needle at rest and dropped from a certain height. The droplet is accelerated by gravity and impacts on the horizontal surface perpendicularly. The impact speed, $U_{0}$, is computed from a calibrated height-speed profile. Rain consists of droplets with a maximum diameter $6 \mathrm{~mm}$ [24] falling at their terminal velocity. For a droplet diameter of $2.8 \mathrm{~mm}$, the terminal velocity is about $7 \mathrm{~m} / \mathrm{s}$. In this study however, the impact speed is one of the important control parameters and is varied from $1.35 \mathrm{~m} / \mathrm{s}$ to $4.13 \mathrm{~m} / \mathrm{s}$. The target granular packing consists of polydisperse soda lime glass beads with diameter $d_{g}=70-110 \mu \mathrm{m}$ and specific density $\rho_{g}=2500 \mathrm{~kg} / \mathrm{m}^{3}$. While there are studies considering the effect of the wettability of grains [6, 7], the wettability is not varied in this work, where hydrophilic grains are used. Grains are put into an oven at $90{ }^{\circ} \mathrm{C}$ for at least half hour before experiments, after which we let them cool down to room temperature. Various packing fractions, $\phi_{0}$, are prepared by air fluidization and a tapping device [Fig. 2a]. The initial packing fraction $\phi_{0}$ is computed according to the surface height relative to the container edge before impact. During the impact, the height of the substrate surface, $z(x, y)$, is measured by high-speed laser profilometry with a typical depth resolution of $0.1 \mathrm{~mm} /$ pixel. The impact process is captured by a high speed camera (Photron SA-X2) with a frame rate of 10,000 Hz, of which an experimental image is shown in Fig. 2b. For dynamic analysis, the surface height before impact is taken as the reference $z=0$. We use two laser lines for the profilometry, and, as the surface deforms, the lines are deflected. We translate the deflection into the height function, $z(r)$, where $r$ is the distance to the impact center in the horizontal plane, i.e., $r=\sqrt{\left(x-x_{c}\right)^{2}+\left(y-y_{c}\right)^{2}}$, where $x_{c}, y_{c}$ are 

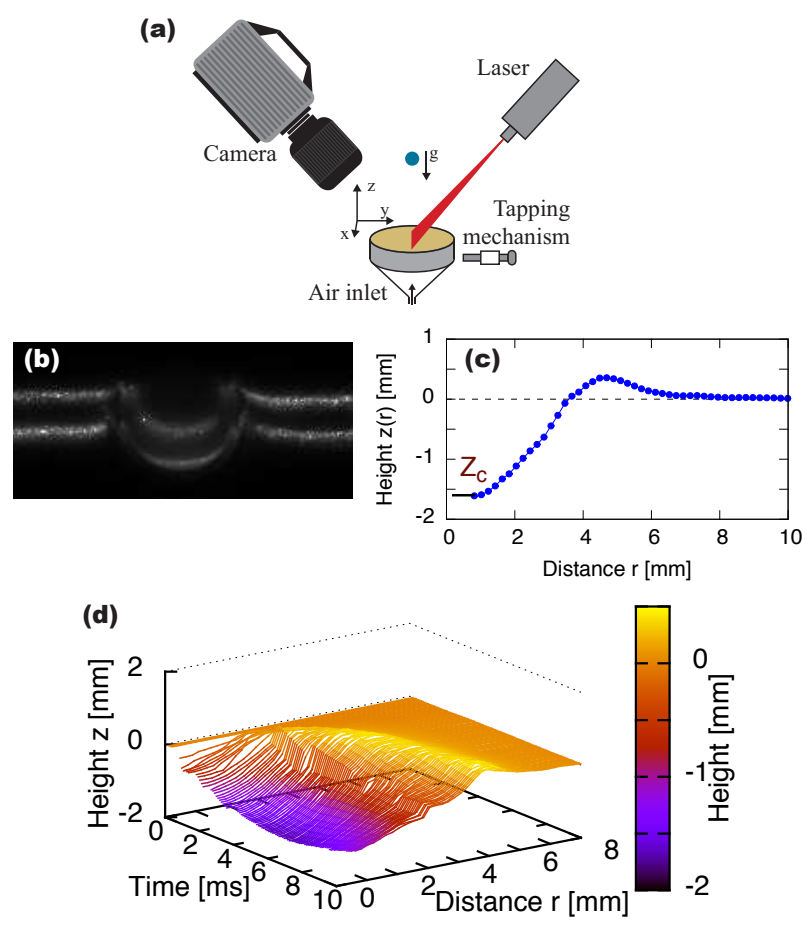

Figure 2. (a) Setup sketch. The height, $z(x, y)$, is measured as a function of coordinates on the horizontal $x y$ plane with laser sheets and a high speed camera. (b) An image taken during the impact, where the droplet is at its maximum deformation. Two laser lines are used for high-speed profilometry, where the deflection of the laser lines indicates the deformation of the target surface. (c) The laser line deflection in (b) is used to reconstruct the height, $z$, as a function of the distance to the impact center, $r$. The dashed line indicates the initial surface height, $z=0$ and the center depth is labeled $Z_{c}$. (d) Time evolution $z(r, t)$ of the crater depth (color coded).

the coordinates of the impact center. Assuming axisymmetry, the impact center is located by determining when the deflections reconstructed from individuals laser lines match. Henceforth, the height function, $z(r)$, used for further analysis is always averaged over the two laser lines. An example of $z(r)$ and its time evolution are illustrated in Fig. 2(c) and (d).

\section{Substrate Deformation}

We use the depth of the crater center, $Z_{c}(t)$ as defined in Fig. 2(c), to characterize the development of the crater. A typical temporal evolution of the crater depth is shown in Fig. 3. In the beginning the crater becomes deeper with time. At 


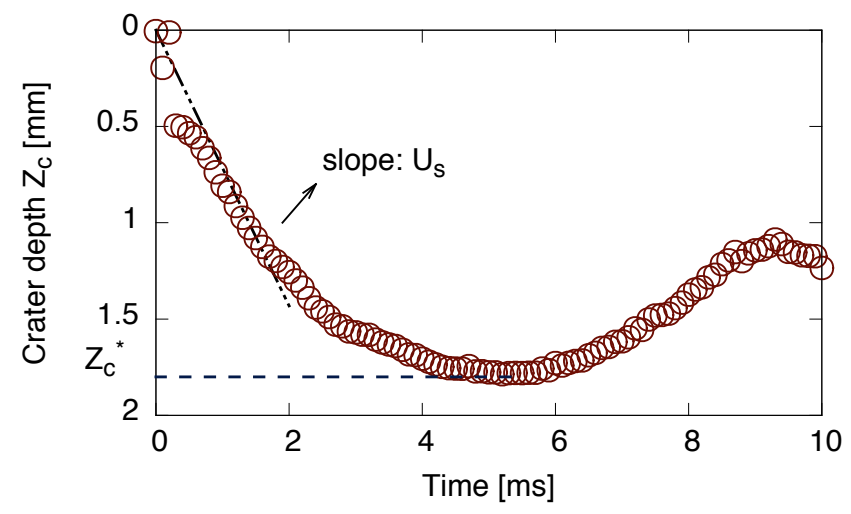

Figure 3. Crater depth versus time. The maximum crater depth is denoted as as $Z_{c}^{*}$. A straight line is fitted on the data points of $t \leq\left(D_{0}+2 Z_{c}^{*}\right) / U_{0}$. The slope of this fit, $U_{s}$, is used to indicate the speed of the deformation. Here, $U_{0}=3.19 \mathrm{~m} / \mathrm{s}$, and $\phi_{0}=0.569$.

a certain moment, the crater reaches its maximum depth $Z_{c}^{*}$, after which the droplet contracts and transports grains mixed with it towards the crater center. An avalanche subsequently occurs at a longer time scale. Both of these effects tend to decrease $Z_{c}$, i.e., make the crater shallower. Here, we focus only on the early stage of $Z_{c}(t)$ and quantify its evolution by measuring two quantities: the initial speed of the deformation, $U_{s}$, and the maximum crater depth, $Z_{c}^{*}$ [25]. The behavior of these two quantities is essential to understand not only the response of the granular substrate, but also the deformation of the droplet and the formation of various crater shapes.

2.1. Deformation speed $U_{s}$. The initial deformation speed $U_{s}$ is determined as the slope of a linear fit of $Z_{c}(t)$ within a time duration $t \leq t_{i m p}$, where $t_{i m p}=$ $\left(D_{0}+2 Z_{c}^{*}\right) / U_{0}$ denotes the impact time scale. [Fig. 3]. The ratio of the impact velocity and this deformation speed is plotted in Fig. 4 for all experiments. This ratio is largely independent of impact speed which implies that it is an inherent property of the granular substrate. On the other hand, the dependence on the packing fraction $\phi_{0}$ indicates a transition around $\phi^{*} \approx 0.585$. * Here, we introduce a simple scenario to elucidate this transition. Upon impact the droplet - with a mass $M_{d}$ - accelerates a certain amount of grains in the vertical direction to the initial deformation speed $U_{s}$, while by vertical momentum conservation the momentum lost by the droplet is proportional to $M_{d}\left(U_{0}-U_{s}\right)$. Thus, the mass of grains accelerated by the droplet is $\left(U_{0}-U_{s}\right) / U_{s}$ times larger than the mass of the droplet. This ratio, plotted in Fig. 4, therefore indicates the amount of substrate material involved in the deformation dynamics and the yield stress of

*Although the measurement of $U_{s}$ is merely the first approximation of the deformation speed, we confirm that $\phi^{*}$ is not altered by reducing the fitting region to half its size. 


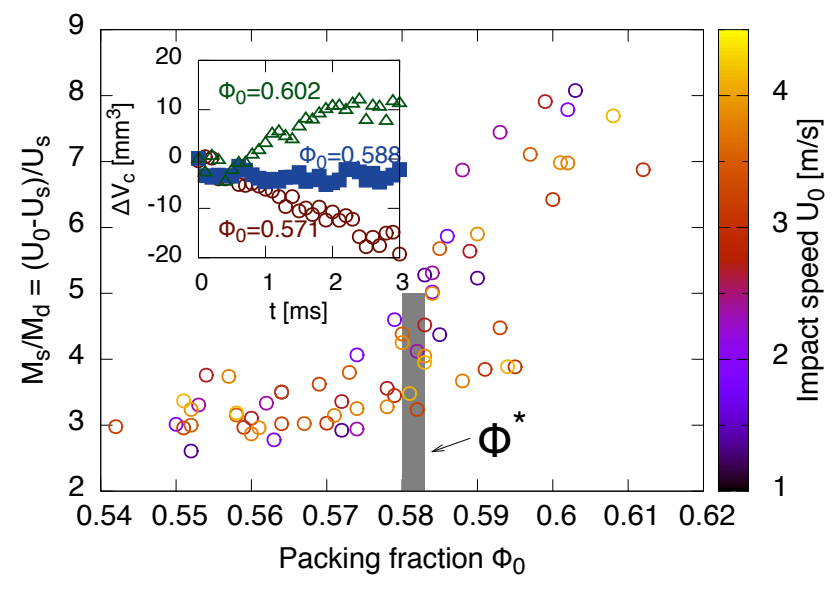

FiguRE 4. Main plot: The ratio of the impact velocity $U_{0}$ and the deformation speed of the substrate $U_{s}$ as a function of packing fraction $\phi_{0}$. The Y-axis, $\left(U_{0}-U_{s}\right) / U_{s}$, indicates the amount of grains involved in the deformation $M_{s}$ normalized by the droplet mass $M_{d}$ (see text), where $U_{s}$ is the speed of the substrate deformation [Fig. 3]. Inset: the volume change of the substrate, $\Delta V_{c}$, for experiments with the same impact speed $U_{0}=3.75 \mathrm{~m} / \mathrm{s}$, but three different packing fractions, $\phi_{0}=0.571,0.588,0.602$, from bottom to top.

the substrate, i.e., the larger this ratio is, the harder the substrate is. With this interpretation, the observed transition at $\phi^{*}$ in Fig. 4 is reminiscent of the penetration force transition which points to the dilatancy transition [26].

The dilatancy transition expresses a peculiar phenomena that shearing a granular packing above a threshold packing fraction results in dilation (i.e., expansion along the perpendicular directions), whereas a loose packing is just compactified under such a perturbation [27]. Though the word 'dilation' describes a change of the volume, previous studies have shown that the dilatancy transition is accompanied by a force response transition [21,22, 26, 28]. As explained above, this force transition feature is already encapsulated by the factor $\left(U_{0}-U_{s}\right) / U_{s}$ representing momentum transfer, and the volume change of the substrate is supposed to give a transition at $\phi^{*}$ as well. We compute the volume change as the integral, $\Delta V_{c}=\pi \int z(r) d r^{2}$, for each frame. The result is plotted in the inset of Fig. 4 for three experiments with different $\phi_{0}$ but the same impact speed $U_{0}$. The volume change is negative (i.e., the substrate compactifies) for the loosest substrate and indeed increases to positive (indicating dilation) with increasing $\phi_{0}$. Based on the above evidence we define a critical packing fraction $\phi^{*} \approx 0.585$ The grains

\footnotetext{
${ }^{\dagger}$ Note that these measurements pertain to the initial deformation of the bed, i.e., before liquidgrain mixing becomes important. The dilation and its transition reported here are inherent properties of granular packings, independent of the intruder properties. This is different to the
} 
underneath the droplet intruder are forced to rearrange to $\phi^{*}$ during the impact whatever the initial packing fraction $\phi_{0}$ is. This value will be used to model the mixing between liquid and grains.

2.2. Maximum crater depth $Z_{c}^{*}$. In contrast to Fig. 4, the maximum crater depth, $Z_{c}^{*}$ plotted in Fig. 5 a, depends on both the initial packing fraction, $\phi_{0}$, and the impact speed of the droplet, $U_{0}$. It has been shown above that a dense substrate is more difficult to deform, therefore it should come as no surprise to see that the maximum crater depth $Z_{c}^{*}$ decreases with $\phi_{0}$. The increase of $Z_{c}^{*}$ with the impact speed $U_{0}$ is also anticipated: a higher impact energy generates a deeper crater.

Studies on solid intruder impact on a granular substrate [17, 18, 20] can help to further understand the impact speed dependence. For a crater created by a solid object, two scaling laws of its depth with impact energy are suggested based on how the kinetic energy, $E_{k}$, is dissipated [17]. Assuming that plastic deformation is the most dissipating process, the crater volume scales with the kinetic energy, which yields $Z_{c}^{*} \sim E_{k}^{1 / 3}[18,20]$. On the other hand, if material ejection absorbs most of the impact energy, then the converted gravitational energy leads to $Z_{c}^{*} \sim E_{k}^{1 / 4}$. The volume change in Fig. 4 implies strong plastic deformations during the impact. However, in the case of droplet impact, not only the substrate but also the intruder dissipates the impact energy via deformations. To apply the above arguments, we therefore first need to decide upon the distribution of $E_{k}$ between the droplet and the granular packing.

The droplet experiences a deceleration force. This force does work on both the droplet and the granular target along the total displacement, $\frac{1}{2} D_{0}+Z_{c}^{*}$. This work transforms the impact energy $E_{k}=M_{d} U_{0}^{2} / 2$ into other energy forms, such as surface energy of the droplet and dissipation inside the substrate. Out of the total displacement, $\frac{1}{2} D_{0}$ is the contribution of the droplet deformation, while $Z_{c}^{*}$ is that of the substrate deformation. Such a force, $E_{k} /\left(\frac{1}{2} D_{0}+Z_{C}^{*}\right)$, indicates the average interaction between droplet and the substrate, from which we can estimate the energy absorbed by the droplet $E_{d}$ and that absorbed by the substrate $E_{s}$ as the work done by this interaction force to deform the droplet and substrate respectively:

$$
E_{d}=\frac{D_{0}}{D_{0}+2 Z_{c}^{*}} E_{k} ; E_{s}=\frac{Z_{c}^{*}}{D_{0} / 2+Z_{c}^{*}} E_{k}
$$

This energy distribution is estimated using the average force between the intruder and the target. While it has been shown that the impact force between a droplet and a solid surface is time dependent [15,29], the subsequent analysis justifies Eq. 1 by hindsight.

dilation measured from the final crater volume [3], where the residue of the liquid-grain mixture plays a very important role. 


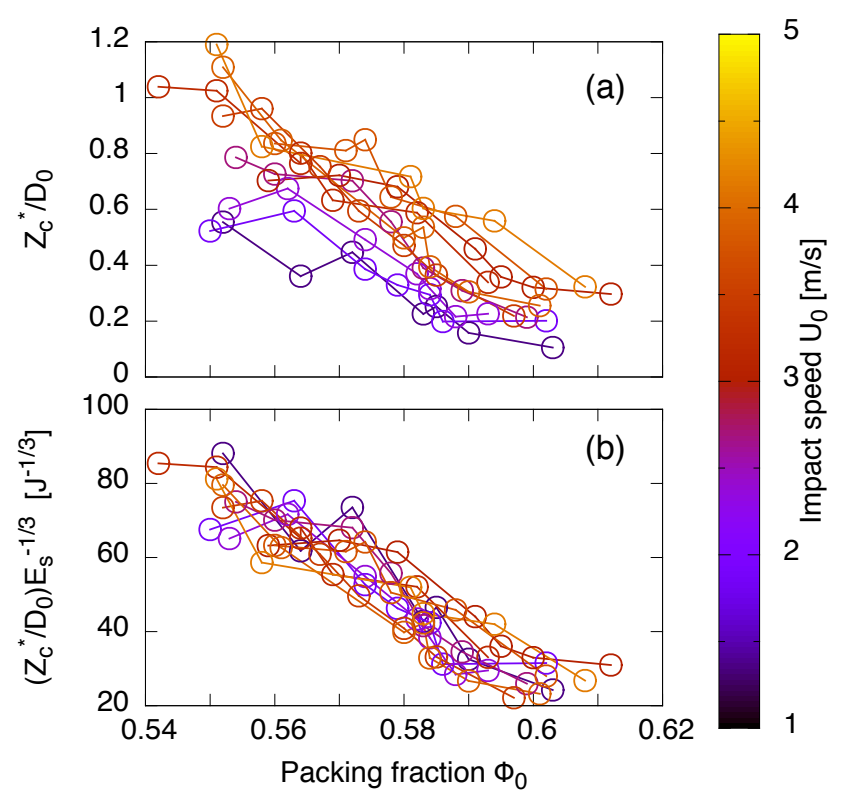

FiguRE 5. (a): Maximum crater depth $Z_{c}^{*}$ (non-dimensionalized with the droplet radius $D_{0}$ ) versus packing fraction $\phi_{0}$. The impact velocity, $U_{0}$, is color coded. The dependence on $\phi_{0}$ is stronger than that on $U_{0}$. (b): Maximum crater depth scaled with the cubic root of the energy transferred into the substrate, $E_{s}$, again versus $\phi_{0}$. See text and Eq. 1 for details.

With measured $Z_{c}^{*}$ and $E_{k}$, the energy distributed into the substrate $E_{s}$ can be computed for all experiments. We find that $Z_{c}^{*} \propto E_{s}^{1 / 3}$ is the better relation to collapse the dependence on $U_{0}$ [Fig. 5 b]. For a given $\phi_{0}$, a power law fit gives $Z_{c}^{*} \propto E_{s}^{\alpha}$ with $\alpha=0.33 \pm 0.04$. This scaling suggests plastic deformation as the main cause of energy dissipation, which stands to reason. We will show that the kinetic energy distribution in Eq. 1 has essential consequences to the droplet deformation and the crater morphology as well.

\section{Maximum Droplet diameter $D_{d}^{*}$}

In contrast to solid object impact, the droplet deforms during impact [2, 4, 5]. This droplet deformation changes the contact area between liquid and grains, which will turn out to play an important role in determining the crater morphology. In this section we focus on the maximum spreading of the droplet.

For most of our experiments, the droplet contracts after spreading, which implies that surface tension is the dominant stopping force of droplet deformation. In this regime, the Weber number $\mathrm{We}=\rho_{l} D_{0} U_{0}^{2} / \sigma$ is the expected relevant dimensionless parameter measuring the relative importance of the kinetic energy of the impact 


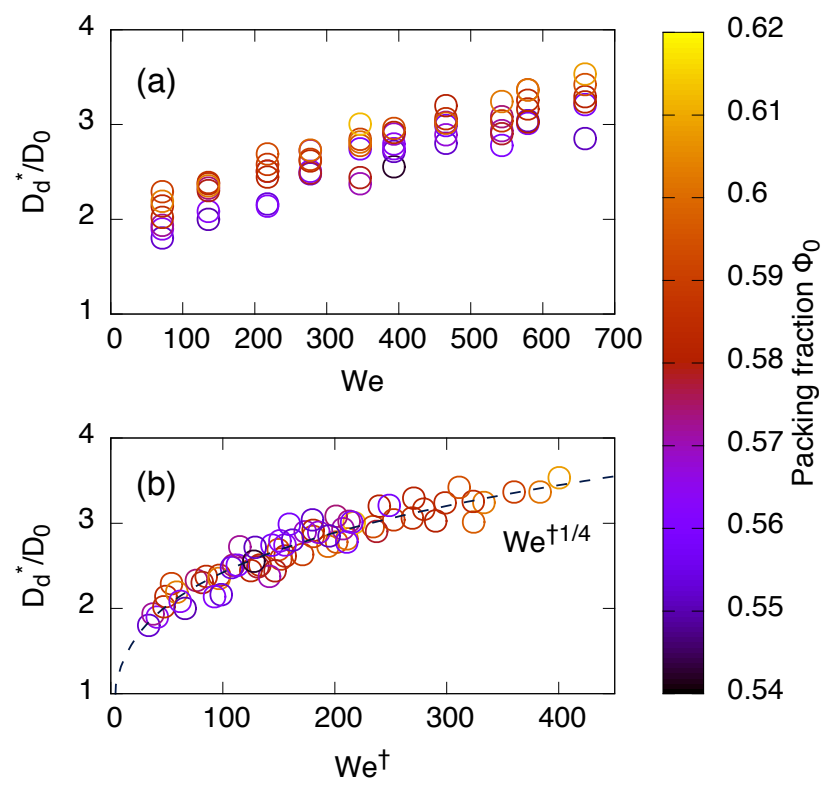

FiguRE 6. Maximum droplet deformation $D_{d}^{*}$ normalized with the initial droplet diameter $D_{0}$ versus Weber number $W e=\rho_{l} D_{0} U_{0}^{2} / \sigma$ in (a) and the effective Weber number $W e^{\dagger}=D_{0} /\left(D_{0}+2 Z_{c}^{*}\right) W e$ in (b), where $Z_{c}^{*}$ is the maximum crater depth [Fig. 3]. Packing fraction is color coded. The dashed line in (b) indicates $D_{d}^{*} / D_{0} \propto$ $W e^{\dagger 1 / 4}$.

droplet and the surface energy. Here, $\rho_{l}$ is the density of the liquid and $\sigma$ is its surface tension. Previous studies about droplet impact on a solid substrate have proposed two models for maximum diameter, $D_{d}^{*}$, that is reached by the droplet. One is based on energy conservation argument where the kinetic energy is converted to the surface energy. This model results in a scaling $D_{d}^{*} / D_{0} \propto \mathrm{We}^{1 / 2}$ 30]. The other more recent model balances the inertial force with the surface tension and suggests $D_{d}^{*} / D_{0} \propto \mathrm{We}^{1 / 4}$ [14]. In the latter case significant amount of the impact energy is dissipated into internal degrees of the droplet.

In Fig. 6a, the maximum droplet diameter in our experiments is plotted against the Weber number $W e$ for different packing fractions. We observe that data with the same impact speed $U_{0}$ are scattered due to the variation in packing fraction, where a denser packing typically generates a larger droplet deformation. This can be understood from the dependence of the substrate deformation on the initial packing fraction [Fig. 5] together with Eq. 1. It has been shown that a denser packing deforms less, thus more energy is transferred into the droplet resulting in a larger spreading. In the energy conservation model [30], We needs to be replaced with an effective Weber number $W e^{\dagger}=D_{0} /\left(D_{0}+2 Z_{c}^{*}\right) W e$. Furthermore, during 
the impact the droplet experiences an average deceleration force with magnitude $\sim$ $E_{d} / \frac{1}{2} D_{0}$ [Eq. 1]. Reproducing the argument in the force balance model introduced in ref. 14, the droplet deforms to balance such a deceleration, which yields a scaling with $W e^{\dagger}$ as well. Therefore, we suggest to use $W e^{\dagger}=\frac{D_{0}}{D_{0}+2 Z_{c}^{*}} W e$ for the impact on a deformable substrate, where $Z_{c}^{*}$ characterizes the deformation of the substrate.

When the maximum droplet deformations are plotted versus effective Weber number in Fig. 6b, we find that the data collapse onto a master curve. A power law fit gives $W e^{\dagger^{0.250 \pm 0.012}}$, which implies that our experiments are well described by the force balance model of Clanet et al [14. Previous studies on this topic rather used the traditional Weber number and reported smaller scaling exponents no larger than $1 / 5[2,5]$. This had been interpreted either as a viscous effect [5] or as a result of the density ratio of the liquid and sand [2]. However, liquids with the same viscosity and density but different surface tension scale differently [5]. We speculate that, other than viscosity, the impact energy distribution in Eq. 1 may be helpful to understand those inconsistent observations in the literature. The mechanism leading to Eq. 1 is that the impact energy $E_{k}$ is distributed according to the relative stiffness of the intruder and the target, i.e., the balance goes always towards the most easy deformation. The extreme case is that either the intruder or the target is undeformable, in which case the energy quickly transfers into the deformable medium. When both intruder and target are deformable, the impact energy dissipates more into the 'softer' one. For instance, while impacting on the same substrate, a liquid droplet with a smaller surface tension is easier to deform, and in consequence the substrate behaves more solid-like, and $W e^{1 / 4}$ is more likely to be recovered. This could explain the different scaling exponents found for liquids that only differ in surface tension in ref. 5. The computation of Eq. 1 requires the maximum crater depth, $Z_{c}^{*}$, which is measured for the first time in the present experiments.

\section{LIQUID-GRAINS MIXTURE AND CRATER MORPHOLOGY}

Until so far we considered the deformation of the target and that of the intruder separately. However, they are 'miscible' as well, i.e., the liquid that composes the droplet penetrates into the granular substrate. This penetration results in a liquidgrain mixture which needs to be taken into account in order to understand the crater morphology [4,5,9]. By changing impact speed $U_{0}$ and the initial packing fraction $\phi_{0}$ the crater morphology is found to vary systematically. According to the residue shape the observed craters are categorized into three groups: doughnut, truffle, and pancake [Fig. 1]. The residue consists of a mixture of liquid and grains, which is referred to as 'liquid marble' in ref. 9. The discrimination between doughnut and truffle is vague, as while changing the impact parameters one shape seems to be continuously transformed into the other, whereas the transition from 
truffle to pancake is more abrupt. In this section, a model about the liquid penetration is established together with the knowledge already obtained in the previous sections. We will use this model to explain the various crater morphologies.

The doughnut and truffle residues are formed from the grains mixed with the liquid which are transported towards crater center by the droplet contraction. If the penetration during the impact is little, after the contraction, pure liquid concentrates at the crater center surrounded by the mixture of liquid and grains. After a few hundreds of milliseconds, the liquid in the center penetrates into the substrate due to gravity and capillary force leaving a dimple, i.e., creating a doughnut residue. More penetration results in a smaller amount of pure liquid at the center, such that the residue gradually becomes a truffle. With even more penetration the droplet is hardly able to contract, leaving a flat residue, the pancake shape. Therefore, knowing the amount of penetration of liquid between grains is crucial to understand the morphology phase diagram.

The transition from doughnut to truffle is continuous, while a sharper transition between truffle and pancake is observed. We estimate the volume of the liquidgrain mixture to characterize the penetration amount and define a threshold volume $V^{*}$ to separate doughnut/truffle and pancake regime. When this threshold mixture volume, $V^{*}$, is reached, the droplet loses the high curvature edge that promotes contraction. This happens when the volume of the mixture in the substrate, $V^{*}$, equals the volume of pure liquid above the substrate, $V_{l}-V^{*}(1-\phi)$, where $V_{l}$ is the initial droplet volume [Fig. 7 inset]:

$$
V^{*}=\frac{V_{l}}{2-\phi} .
$$

If the time scale to reach this critical mixing volume, $t_{m i x}$, is shorter than the impact time scale $t_{i m p}=\left(D_{0}+2 Z_{c}^{*}\right) / U_{0}$, the droplet contraction is suppressed, and pancake shapes are observed. Otherwise, the droplet is able to contract, mixed with grains, and forms a doughnut/truffle residue.

To quantitatively examine the above picture, we need to formulate the mixing progress. Describing the granular substrate as a porous medium, we start with Darcy's law:

$$
\frac{Q}{A} \approx \frac{\kappa P}{\mu L}
$$

Here, the permeability of the granular packings, $\kappa$, is defined by Carman-Kozeny relation $\kappa=(1-\phi)^{3} d_{g}^{2} /\left(180 \phi^{2}\right)$, where $Q$ is the volume flux into the porous substrate, $P$ the driving pressure, $\mu$ the dynamic viscosity of the liquid, $L$ the penetration depth of the liquid into the porous substrate, and $A$ the contact area. In Eq. 3 we estimate the pressure gradient inside the sand as $P / L$. Furthermore, the conservation of liquid volume calls for

$$
\frac{Q}{A}=(1-\phi) \frac{d L}{d t}
$$


Here, we only consider $L$ as a function of time. The total liquid volume penetrating into the substrate is given by $(1-\phi) A L$.

Combining Eq. 3 and Eq. 4, the penetration depth $L$ is solved as $L(t)=$ $\sqrt{2 P \kappa /(\mu(1-\phi)) t}$. From here, one can define the time scale for which the volume of the mixture reaches the critical volume, $A L\left(t_{\text {mix }}\right)=V^{*}$ :

$$
t_{m i x}=\left(\frac{V^{*}}{A}\right)^{2} \frac{(1-\phi) \mu}{2 \kappa P} .
$$

To apply Eq. 5 a few quantities need to be evaluated and explained. The contact area is estimated as $A=\pi D_{d}^{* 2} / 4$, i.e., the contact area at the measured maximum spreading diameter. The packing fraction $\phi$ is evaluated by the critical packing fraction $\phi^{*}$ rather than the initial packing fraction $\phi_{0}$ as explained in Fig. 4. The last missing piece is the driving pressure $P$. There are three candidates: the gravitational pressure, the inertial pressure, and the capillary pressure caused by the hydrophilicity of the grains that tries to pull the liquid into the bed. Since the droplet diameter is at the magnitude of the capillary length, gravity cannot significantly deform the droplet. The large droplet deformation shown in Fig. 6 indicates that the inertial pressure is much larger than gravity. A simple experiment of zero impact speed, in which we determine how fast the droplet is absorbed by the bed, also indicates that for the used grains capillary pressure is at least two order of magnitude smaller than the inertial pressure. Therefore it is justified to consider the inertial pressure as the driving pressure. In the analysis of maximum droplet deformation and the introduction of the effective Weber number $W e^{\dagger}$ it was already implied that the average deceleration force experienced by the droplet equals $E_{d} / \frac{1}{2} D_{0}$ [Eq. 1]. The inertial pressure is evaluated in consequence as:

$$
P=\frac{E_{d}}{\frac{1}{2} D_{0} A}=\frac{M_{l} U_{0}^{2}}{A\left(D_{0}+2 Z_{c}^{*}\right)} .
$$

With all the quantities in Eq. 5 now defined, we can finally compare the time scales, $t_{i m p}$ and $t_{m i x}$, in Fig. 7.

Three features from the experimental phase diagram are recovered in Fig. 7 (i) the transition is dominated by impact speed; (ii) a denser packing induces more mixture; and (iii) the expected transition discriminating doughnut/truffle and pancake morphology around $U_{0} \approx 3.2 \mathrm{~m} / \mathrm{s}$ is indeed indicated by the condition $t_{i m p}=t_{\text {mix }}$. We emphasize that the inertial pressure driving penetration during the droplet spreading is considered to dominate the crater morphology transition, rather than gravity during the recession as was suggested in ref. 4. Therefore, the reference time scale is the characteristic period of the inertial pressure, $t_{i m p}$, rather than the contact time scale $\sim \sqrt{\rho_{l} D_{0}^{2} / \sigma}[9$. Finally, it is worthy to note that according to Eq. 5 and Eq. 6 the ratio of the time scales $t_{i m p} / t_{m i x}$ is independent of the substrate deformation $Z_{c}^{*}$. To apply and check the model, only $D_{d}^{*}$ needs to be measured. 


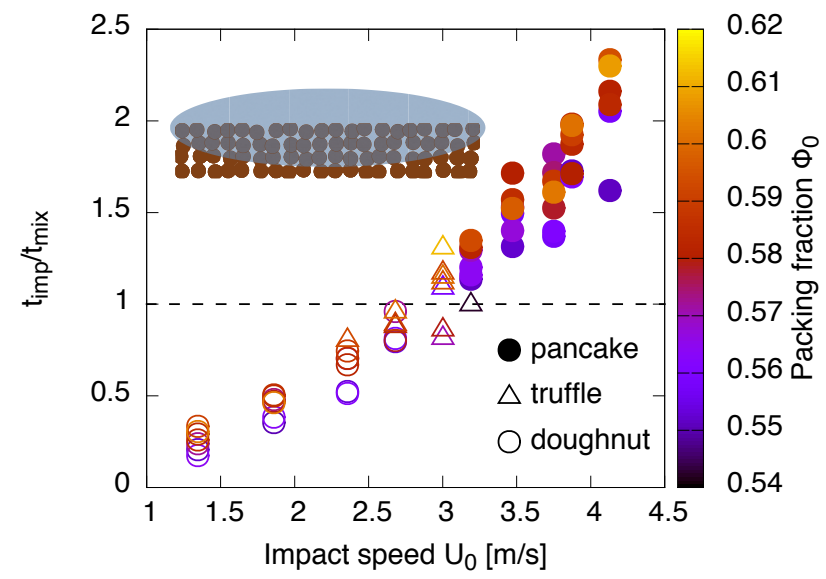

Figure 7. Phase diagram of the crater morphology. On the yaxis we plot the ratio between two time scales, namely the impact time scale $t_{i m p}$ and the mixing time $t_{m i x}$. The horizontal dashed line indicates a ratio of 1 , where the transition is expected. On the $\mathrm{x}$ axis we have the impact speed $U_{0}$. The various crater morphologies are labeled with different symbols (see legend), whereas the color of the symbols indicate initial packing fraction. The inset shows a schematic of the droplet for which $V=V^{*}$ at maximum expansion.

\section{Conclusion}

In this paper, we study the deformation of the droplet and the granular packing during impact. The deformation of the granular substrate decreases with the initial packing fraction [Fig. 5], however, the deformation speed exhibits evidence of dilation and defines a critical packing fraction $\phi^{*} \approx 0.585$ in Fig. 4. The substrate deformation introduces an impact energy distribution, Eq. 1, between the intruder and the target. An effective Weber number, $W e^{\dagger}$, is defined accordingly, and the droplet maximum deformation, $D_{d}^{*}$, is shown to be consistent with a scaling law $D_{d}^{*} \sim W e^{\dagger^{1 / 4}}$ in Fig. 6, which suggests a scenario where surface tension balances inertial pressure. Finally, based on the results of $\phi^{*}$ and $D_{d}^{*}$, a model is established to describe the penetration of the liquid into the substrate. This model evaluates the competition between the penetration time and the impact time, from which it is able to explain the observed morphology transition between the doughnut/truffle and the pancake crater shapes [Fig. (7). The energy distribution as given in Eq. 1, which is essential to understand the deformation of the target and the intruder, is estimated with the average interaction force. Its validation needs time resolved measurement of the interaction force.

We acknowledge the discussion with $\mathrm{X}$. Cheng. This project is supported by FOM and NWO through a VIDI Grant No. 68047512. 


\section{REFERENCES}

[1] Hiroaki Katsuragi. Morphology Scaling of Drop Impact onto a Granular Layer. Phys Rev Lett, 104(21):218001, May 2010.

[2] J.O. Marston, S.T. Thoroddsen, W.K. Ng, and R.B.H. Tan. Experimental study of liquid drop impact onto a powder surface. Powder Technol, 203(2):223-236, November 2010.

[3] Hiroaki Katsuragi. Length and time scales of a liquid drop impact and penetration into a granular layer. J Fluid Mech, 675:552-573, May 2011.

[4] G. Delon, D. Terwagne, S. Dorbolo, N. Vandewalle, and H. Caps. Impact of liquid droplets on granular media. Phys Rev E, 84(4):046320, October 2011.

[5] E Nefzaoui and O Skurtys. Impact of a liquid drop on a granular medium: Inertia, viscosity and surface tension effects on the drop deformation. Experimental Thermal and Fluid Science, 41:43-50, 2012.

[6] J. O. Marston, Y. Zhu, I. U. Vakarelski, and S. T. Thoroddsen. Deformed liquid marbles: Freezing drop oscillations with powders. Powder Technol, 228:424-428, September 2012.

[7] C. A. E. Hamlett, S. Atherton, N. J. Shirtcliffe, G. McHale, S. Ahn, S. H. Doerr, R. Bryant, and M. I. Newton. Transitions of water-drop impact behaviour on hydrophobic and hydrophilic particles. Eur J Soil Sci, 64(3):324-333, June 2013.

[8] Edward J. Long, Graham K. Hargrave, James R. Cooper, Ben G. B. Kitchener, Anthony J. Parsons, Caspar J. M. Hewett, and John Wainwright. Experimental investigation into the impact of a liquid droplet onto a granular bed using three-dimensional, time-resolved, particle tracking. Phys Rev E, 89(3):032201, March 2014.

[9] Runchen Zhao, Qianyun Zhang, Hendro Tjugito, and Xiang Cheng. Granular impact cratering by liquid drops: Understanding raindrop imprints through an analogy of asteroid strikes. Proc Natl Acad Sci U S A, 112:342-347, July 2015.

[10] Heather N. Emady, Defne Kayrak-Talay, William C. Schwerin, and James D. Litster. Granule formation mechanisms and morphology from single drop impact on powder beds. Powder Technol, 212(1):69-79, September 2011.

[11] Heather N. Emady, Defne Kayrak-Talay, and James D. Litster. A regime map for granule formation by drop impact on powder beds. AIChE J, 59(1):96-107, January 2013.

[12] Heather N Emady, Defne Kayrak-Talay, and James D Litster. Modeling the granule formation mechanism from single drop impact on a powder bed. J Colloid Interface Sci, 393:36976, March 2013.

[13] M Rein. Phenomena of liquid drop impact on solid and liquid surfaces. Fluid Dyn Res, 12:61-93, 1993.

[14] Christophe Clanet, Cédric Béguin, Denis Richard, and David Quéré. Maximal deformation of an impacting drop. J Fluid Mech, 517:199-208, September 2004.

[15] Jens Eggers, Marco a. Fontelos, Christophe Josserand, and Stephane Zaleski. Drop dynamics after impact on a solid wall: Theory and simulations. Phys Fluids, 22(6):062101, 2010.

[16] Heinrich Jaeger, Sidney Nagel, and Robert Behringer. Granular solids, liquids, and gases. Rev Mod Phys, 68(4):1259-1273, October 1996.

[17] Joseph C. Amato. Crater formation in the laboratory: An introductory experiment in error analysis. Am J Phys, 66(2):141, 1998.

[18] J. Uehara, M. Ambroso, R. Ojha, and D. Durian. Low-Speed Impact Craters in Loose Granular Media. Phys Rev Lett, 90(19):194301, May 2003.

[19] Detlef Lohse, Raymond Bergmann, René Mikkelsen, Christiaan Zeilstra, Devaraj van der Meer, Michel Versluis, Ko van der Weele, Martin van der Hoef, and Hans Kuipers. Impact on Soft Sand: Void Collapse and Jet Formation. Phys Rev Lett, 93(19):198003, November 2004 . 
[20] Hiroaki Katsuragi and Douglas J. Durian. Unified force law for granular impact cratering. Nat Phys, 3(6):420-423, April 2007.

[21] Paul Umbanhowar and Daniel I. Goldman. Granular impact and the critical packing state. Phys Rev E, 82(1):010301, July 2010.

[22] Nick Gravish, Paul B. Umbanhowar, and Daniel I. Goldman. Force and Flow Transition in Plowed Granular Media. Phys Rev Lett, 105(12):128301, 2010.

[23] J C Ruiz-Suárez. Penetration of projectiles into granular targets. Rep Prog Phys, 76(6):066601, June 2013.

[24] Emmanuel Villermaux and Benjamin Bossa. Single-drop fragmentation determines size distribution of raindrops. Nat Phys, 5(9):697-702, September 2009.

[25] When the droplet reaches its maximum spreading diameter, its average thickness $h=$ $(2 / 3) D_{0}\left(D_{0} / D_{\max }\right)^{2}$. According to the geometric configuration of the experiments, the depth difference introduced by this thickness is $0.37 h$. For the smallest $U_{0}$, the least spreading case, where the refraction introduces the largest error, this estimation gives a height difference of $0.14 \mathrm{~mm}$, which is close to the depth resolution of the laser profilometry system. Moreover, this refraction error is well below the smallest measured $Z_{c}^{*}$ of $0.5 \mathrm{~mm}$. Since the center of the impact droplet is even thinner than $h$ as estimated above, the influence on the maximum center depth measurement would be even weaker in practice.

[26] Matthias Schröter, Sibylle Nägle, Charles Radin, and Harry L Swinney. Phase transition in a static granular system. Europhys Lett, 78(4):44004, May 2007.

[27] Pa Thompson and Gs Grest. Granular flow: Friction and the dilatancy transition. Phys Rev Lett, 67(13):1751-1754, September 1991.

[28] J.-F. Métayer, D. J. Suntrup III, C. Radin, H. L. Swinney, and M. Schröter. Shearing of frictional sphere packings. Europhys Lett, 93(6):64003, March 2011.

[29] Dan Soto, Aurélie Borel De Larivière, Xavier Boutillon, Christophe Clanet, and David Quéré. The force of impacting rain. Soft matter, 10(27):4929-34, July 2014.

[30] T. Bennett and D. Poulikakos. Splat-quench solidification: estimating the maximum spreading of a droplet impacting a solid surface. J Mater Sci, 28(4):963-970, 1993. 